\title{
Forging a new path: an endoscopic jejunojejunostomy
}

A 74-year-old man with a history of T2N1 gastric adenocarcinoma who underwent a total gastrectomy with a Roux-en-Y reconstruction presented to our institution with dysphagia. Since his surgery, the patient had several presentations to multiple area hospitals with the complaint of dysphagia to solids with vomiting, and what appeared to be intermittent food bolus obstructions. He underwent several dilations of his esophagojejunal anastomosis without improvement of his symptoms, and in fact it seemed that the greatest issue was not a stricture of the anastomosis, but rather twisting of the jejunal limbs making the blind limb the dominant lumen.

This was confirmed with an esophagram, which showed delayed passage of contrast at the esophagojejunal level, with the contrast emptying into the blind jejunal limb. Contrast was then expelled through a narrow and angulated proximal efferent alimentary limb. Inspired by recent off-label use of lumen-apposing metal stents (LAMS) [1,2], we proposed fistulization of the two jejunal limbs using a LAMS. A guidewire was advanced into the efferent limb, followed by the endoscope, which was then used to flood the lumen of the efferent limb with methylene blue, Omnipaque, and saline. A linear echoendoscope was then advanced into the blind limb and used to identify the distended, fluid-filled efferent limb and guidewire. A 15-mm LAMS was then deployed, fistulizing the two limbs (\Video 1).

An esophagram 24 hours after the procedure showed improved flow of ingested material into the efferent jejunal limb, and at 2-week follow-up the patient was doing well on a full liquid diet without any further dysphagia. The patient continues to do well and gain weight at 2 months post-procedure. This case high-

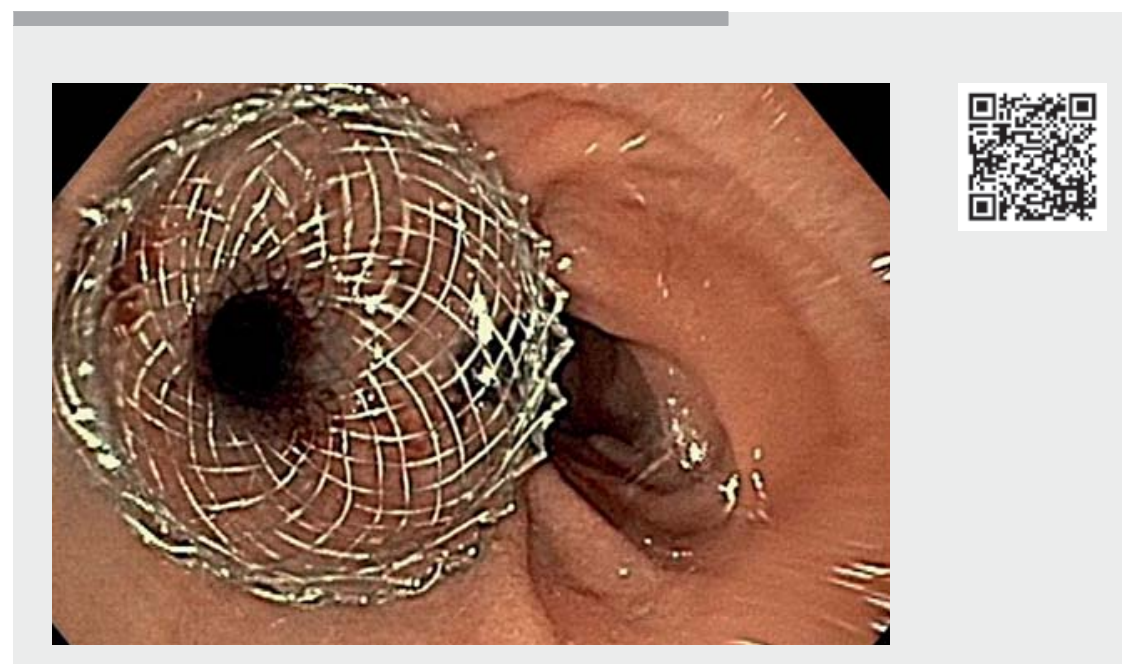

Video 1 An endoscopic jejunojejunostomy. Echoendoscopic views with depth displayed in centimeters on the left side of the image.

lights another practical application of LAMS in helping bypass functional obstructions to improve a patient's symptoms and quality of life.

Endoscopy_UCTN_Code_TTT_1AS_2AG

\section{Competing interests}

The authors declare that they have no conflict of interest.

\section{The authors}

Daniel Hogan ${ }^{1}$, Maheep Sangha ${ }^{1}$, Paul Lee ${ }^{2}$, Evin McCabe ${ }^{1}$, Petros Benias'

1 Division of Gastroenterology, Lenox Hill Hospital, Northwell Health, New York, NY

2 Division of Cardiovascular and Thoracic Surgery, Lenox Hill Hospital, Northwell Health, New York, NY

\section{Corresponding author}

Daniel Hogan, DO

Division of Gastroenterology, Lenox Hill Hospital, Northwell Health, 100 E. 77th, St., 2nd, Floor, Lachman Pavilion, New York, NY 10075, USA

DHogan3@Northwell.edu

\section{References}

[1] Sharma P, McCarty TR, Chhoda A et al. Alternative uses of lumen apposing metal stents. World J Gastroenterol 2020; 26: 2715-2728

[2] Trindade A, Sejpal D, Benias PC. Palliation of malignant pyloric stenosis using a lumenapposing metal stent. Clin Gastroenterol Hepatol 2020; 17: A18

Bibliography

Endoscopy 2021; 53: E461

DOI 10.1055/a-1346-7678

ISSN 0013-726X

published online 4.2.2021

(c) 2021. Thieme. All rights reserved.

Georg Thieme Verlag KG, Rüdigerstraße 14, 70469 Stuttgart, Germany 